5. O’Mahony C, Jichi F, Pavlou M, Monserrat L, Anastasakis A, Rapezzi C, et al. A novel clinical risk prediction model for sudden cardiac death in hypertrophic cardiomyopathy (HCM risk-SCD). Eur Heart J. 2014;35:2010-20.

https://doi.org/10.1016/j.jtcvs.2017.12.040

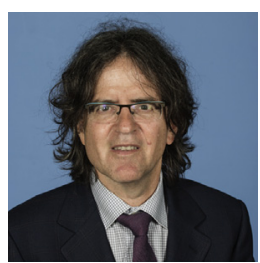

A DEDICATED HEART TEAM IS NEEDED TO MAXIMIZE THE TREATMENT OF PATIENTS WITH HYPERTROPHIC CARDIOMYOPATHY

Reply to the Editor:

The letter by Dr Srilakshmi is a thoughtful representation of the current challenges we are all facing in treating patients with hypertrophic cardiomyopathy (HCM). These patients, when diagnosed or become symptomatic, pose a unique challenge in achieving better control of symptoms and minimizing the risk of sudden death. The issue raised by Dr Srilakshmi of risk prediction to better guide the use of an automatic implantable cardioverter-defibrillator is an interesting one. However, it remains theoretical only because no risk prediction model is perfect, let alone one with such a small number of patients available. I agree that the indications to use automatic implantable cardioverter-defibrillators to minimize the risk of sudden death due to malignant ventricular arrhythmia may not always be clear, but the risk-benefit considerations are well accepted among the medical community and the patients. In the case series published by Drs Nguyen and Schaff from the Mayo Clinic, it was clearly demonstrated that surgical treatment can be safe and effective in many cases and especially for those with midventricular obstruction and malignant arrhythmias.

Currently, the approach to HCM in most centers does not involve a true heart team with a thorough discussion and planning of each case. It is clear that there needs to be improved communication and refinement in the clinical approach to such patients. One of the challenges that most centers face is the small number of patients treated and the lack of significant clinical experience, especially when it comes to midventricular lesions. In general, the surgical community is not familiar with the surgical approaches appropriate for this subgroup of patients, mainly because of the rarity of HCM. As a subspecialty, it is imperative to recognize that a well-skilled surgical team can perform high-risk procedures with outstanding outcomes. Therefore, there is a need to approach HCM as a team to better understand the genetics, the heterogeneous clinical presentation, and all the different treatment options, including surgery. That being said, a good and reliable risk algorithm is
Author has nothing to disclose with regard to commercial support.

always necessary and should be incorporated in our clinical practice if proven to improve patient outcomes.

Niv Ad, MD

Department of Cardiovascular and Thoracic Surgery

West Virginia University

Morgantown, WVa

https://doi.org/10.1016/j.jtcvs.2018.02.075

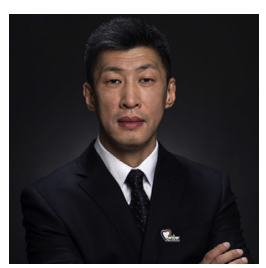

\section{STRAIGHT DEEP \\ HYPOTHERMIC CIRCULATORY \\ ARREST: CLING TO OLD FASHION OR NOT?}

To the Editor:

We read with great interest the study by Damberg and colleagues ${ }^{1}$ showing excellent results after aortic surgery with straight deep hypothermic circulatory arrest (DHCA) without cerebral perfusion. Antegrade cerebral perfusion (ACP) was not performed to avoid potential air/particulate embolism and hypoperfusion or hyperperfusion. They demonstrated that straight DHCA (DHCA time within 50 minutes) was a safe and effective method of brain protection in patients at their center, associated with favorable short-term mortality (2.9\%) and stroke rate $(2 \%)$, as well as late survival (estimated 1and 5-year survivals $92.2 \%$ and $81.5 \%$, respectively), which was comparable to outcomes in other cohorts using ACP.

However, we suppose these favorable results could be achieved only at the best hands among selected patients. The generality of their conclusion should be questioned. Under some circumstances, such as patients aged more than 60 years, redo surgery, emergency surgery, dissection, total arch replacement, and junior surgeon at the deep portion of the learning curve, it is reasonable to provide an extra layer of brain protection by ACP.

To the best of our knowledge, adjunctive ACP has been shown to be superior over straight DHCA by single-center cohort studies: a large-scale comparative effectiveness study from the Society of Thoracic Surgeons database ${ }^{2}$ and a meta-analysis. ${ }^{3}$ During circulatory arrest, a sharp decrease of cerebral oxygenation was observed in DHCA compared with ACP, increasing the risk of neurologic complications. ${ }^{4}$ Furthermore, deep 
Authors have nothing to disclose with regard to commercial support.

hypothermia was no longer a must when ACP was used. Keeling and colleagues ${ }^{5}$ compared 2 temperature strategies for total aortic arch replacement with ACP (moderate hypothermia circulatory arrest [MHCA] vs DHCA). There was no difference in neurologic outcomes or inhospital mortality for the 2 temperature groups. ${ }^{5}$ The perfusion strategy for aortic arch surgery has been evolving. In our institute, MHCA (nasal temperature $25^{\circ} \mathrm{C}$ ) plus ACP is the current practice. The rationale is that MHCA could reduce cardiopulmonary bypass time, which has been clearly demonstrated as a risk factor for worse outcome.

Anecdotally, some surgeons are trying to replace the total aortic arch at a higher temperature (nasal temperature $28^{\circ} \mathrm{C}$ ), requiring a brief lower-body circulatory arrest $(<5$ minutes), with an inflated balloon occluding the opened descending aorta after releasing the frozen elephant trunk. This method allows more time to create a secure end-to-end anastomosis between the graft and the descending aorta, because the brain and lower body are both perfused by cannulas in the femoral artery and right axillary artery.

We agree that there are some aspects of ACP requiring further clarification, such as bilateral/unilateral perfusion, appropriate flow rates, and pressure. We are approaching an era when aortic surgery could be performed with moderate hypothermia, brief circulatory arrest, and hopefully less disturbed physiology. Clinging to the old fashion seems unwise.

\section{Shujie Yan, $M D, P h D$ \\ Bingyang $J i, M D, P h D$ \\ Song Lou, MD, PhD \\ Department of Cardiopulmonary Bypass State Key Laboratory of Cardiovascular Medicine Fuwai Hospital \\ Chinese Academy of Medical Science and Peking Union Medical College Beijing, China}

\footnotetext{
References

1. Damberg A, Carino D, Charilaou P, Peterss S, Tranquilli M, Ziganshin BA, et al. Favorable late survival after aortic surgery under straight deep hypothermic circulatory arrest. J Thorac Cardiovasc Surg. 2017;154:1831-9.

2. Englum BR, He X, Gulack BC, Ganapathi AM, Mathew JP, Brennan JM, et al. Hypothermia and cerebral protection strategies in aortic arch surgery: a comparative effectiveness analysis from the STS Adult Cardiac Surgery Database. Eur J Cardiothorac Surg. 2017;52:492-8.
}

3. Tian DH, Wan B, Bannon PG, Misfeld M, LeMaire SA, Kazui T, et al. A meta-analysis of deep hypothermic circulatory arrest alone versus with adjunctive selective antegrade cerebral perfusion. Ann Cardiothorac Surg. 2013;2: 261-70.

4. Kamenskaya OV, Klinkova AS, Chernyavsky AM, Lomivorotov VV, Meshkov IO, Karaskov AM. Deep hypothermic circulatory arrest vs. antegrade cerebral perfusion in cerebral protection during the surgical treatment of chronic dissection of the ascending and arch aorta. J Extra Corpor Technol. 2017; 49:16-25.

5. Keeling WB, Tian DH, Leshnower BG, Numata S, Hughes GC, Matalanis G, et al Safety of moderate hypothermia with antegrade cerebral perfusion in total aortic arch replacement. Ann Thorac Surg. 2018;105:54-61.

https://doi.org/10.1016/j.jtcvs.2018.01.001

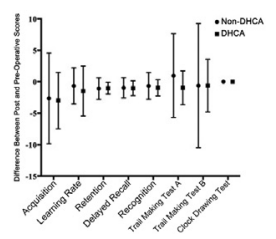

EVERYTHING OLD IS NEW AGAIN

Reply to the Editor:

We are pleased to respond to the letter of Yan and colleagues ${ }^{1}$ commenting on our article $^{2}$ on long-term follow-up of patients undergoing aortic arch operations under straight deep hypothermic circulatory arrest (DHCA). We thank Yan and colleagues ${ }^{1}$ for their kind comments regarding our operative results.

We wish to emphasize that the goal of our article ${ }^{2}$ was not to argue against the use of adjunctive perfusion strategies. Rather, we demonstrated to the surgical community that use of DHCA alone does not hamper the long-term outlook for patients with aortic arch pathology.

In their letter, Yan and colleagues ${ }^{1}$ provide an unbalanced view of available literature on this topic, citing studies supporting adjunctive perfusion but failing to cite studies that support the superiority of DHCA or those showing no benefit from adjunctive perfusion. ${ }^{3-5}$ Although Yan and colleagues ${ }^{1}$ claim superiority of adjunctive antegrade cerebral perfusion, we are not aware of any randomized trials that compare the outcomes of different cerebral protection strategies. Furthermore, most studies, including those mentioned by Yan and colleagues, ${ }^{1}$ typically evaluate heterogeneous groups of patients and may suffer from some degree of outcome bias on the part of the investigators.

We would like to pinpoint certain limitations in the studies that Yan and colleagues ${ }^{1}$ cite as evidence in favor of perfusion:

1. Interestingly, in the study of Englum and associates, ${ }^{6}$ DHCA (without perfusion) was the most commonly used protection strategy, indicating the continued prominent role that DCHA plays in clinical practice today. It makes common sense that the most acutely ill patients (acute dissection, shock) would not undergo the more time-consuming axillary dissection nearly uniformly used with antegrade perfusion strategies. In the study 